\title{
CyA 246
}

National Cancer Institute

\section{Source}

National Cancer Institute. CyA 246. NCI Thesaurus. Code C2101.

A bacterial cell wall mimetic. It is a potent inducer of cytokines and is beginning Phase II trials in combination with chemotherapy for patients with non-small cell lung cancer. 